\title{
Ventilación Mecánica en una Unidad de Neonatología
}

\author{
Dra. M. Soledad Rodriguez T. ${ }^{\text {1; }}$ Dr. Aldo Bancalari M. ${ }^{2}$; Dr. Enzo Pandolfi B. ${ }^{2}$
}

\author{
Mechanical Ventilation in Newborn Infants
}

\begin{abstract}
A retrospective evaluation of mechanical ventilation (MV) in ncwbron jnfant 8 along a seventeen month period in Concepción, Chile, is presented. Hyaline membrare disease (HMD) was present in $25 / 54$ patients and severe neonatal asphixia en 14/54. Complications occured in 31/54 patients, most frecuently extubation, atelectasis and infections. Lethality rates were $59.3 \%(32 / 54)$ for all patients, $60 \%(15 / 25)$ for HND and $71.4 \%(5 / 7)$ for patients born with weights under $1000 \mathrm{~g}$. Twenty two survivors were followed up through ophthatmologic, neurological and pulmonary examinations. No ocular abnormalities were detected but one patient showed evifence of bronchopulmonary displasia and in three there were neutological sequclae.
\end{abstract}

(Key words: Newbron infants. Mechanjeal ventilation, Complications, Lethality. Sequelae).

En los últimos años se ha logrado un descenso importante en la mortalidad neonatal $1,2,3,4,5$, ${ }^{6}$, especialmente en los recién nacidos con ițsuficiencia respiratoria grave, gracias entre otras medidas al uso del ventilador mecátuco (VM). ${ }^{6}$, 7. 8, 9 Sin embargo, el uso del ventilador mecảnico requiere de un constante adiestramiento del equipo profesional que labora en una sala de cuidados intensivos neonatales, por ser un proce. dimiento invasivo y sujeto a múltiples complicaciones, que pueden ser minimizadas por una vigilancia constante y un adecuado entendimiento del manejo de este aparató. ${ }^{10,11,12}$

La ventilación mecánica tiene como objeto primordial dar apoyo a la ventilación hasta que el paciente recupere la capacidad para hacerlo por si mismo. ${ }^{13}$ En el recién nacido estä indicada en casos de severa insuficiencia respiratoria que no responde a otros modos de tratamiento tales como la oxígenoterapia, los estimulantes respira. torios o la presión positiva continua. $13,14,15$, It Hay dos indicaciones básicas para la ventilación a presión positiva intermitente (IPPV). La primera es la hipoventilación e hipercapnia que puede ser secundaria a depresión del sistema nervioso central por asfixia, drogas, hemorragia, infección, apnea del prematuro o a un aumento del trabajo respiratorio, a su vez causado por obstrucción de la via aérea, disminución de la

1. Becada Pedjatría, Facultad de Medicina, Universidad de Concepción.

2. Médico Pediatra, Servicio de Pediatría, Hospital GuiIlermo Grant Benavente, Concepción. distensibilidad pulmonar o ambos $13,14,15,16$ La segunda indicación es una severa hipoxemia por cortocircuito pulmonar. ${ }^{15,16}$ En general hay más de una razón para usar IPPV en el RN. Por ejemplo un neonato prematuro de muy bajo peso puede requerir ventilación al nacimiento por depresión respiratoria secundaria a asfixia y más tarde desarroltar una insuficiencia pulmonar progresiva a causa de una membrana hialina. ${ }^{8,16}$ Luego mientras mejora de su enfermedad pulmonar, un gran cortocircuito de izquierda a derecha a travès de un conducto arterioso permeable frecuentemente ocurre con deterioro de la función pulmonar. ${ }^{17,18}$ Finalmente el mismo RN puede requerir prolongada ventilación a causa de una enfermedad pulmonar crónica o debjda a una depresión central por una hemorragia intracraneana $(\mathrm{HIC})^{8}, 16,19,20,21,22$. Esta secuencia de eventos es muy común en recién nacidos menores đe 28 a 30 semanas de gestación.

La Unidad de Neonatología del hospital Gui. llermo Grant Benavente de Concepción, comenzó e] uso de la ventilación mecánica a principios de 1983. El objetivo del presente estudio fue evaluar la evolución de los recién nacidos sometidos a ventilación mecánica por 24 horas o más.

\section{MATERIAL Y METODO}

Se analizaron los registros de recién nacidos que fueton conectados a ventilación mecánica (VM) en la Unidad de Cuidados Intensivos Neonatales del Servicio de Pediatría del Hospital Guillermo Grant Benavente de Concepción, entre el $1^{\circ}$ de Abril de 1983 al 30 de Septiembre de 1984 (17 meses). En el estudio fueron considerados el peso; la edad gestacional, Apgar al nacer; 
procedencia; duración de la ventilación mecánica; complicaciones; mortalidad global; mortalidad por patología y peso de nacimiento. Se realizó seguimiento a todos los recién nacidos sobrevivientes, mediante fondo de ojo efectuado por an mismo oftalmólogo, examen neurológico y examen pulmonar a través de una o más radjografías de tórax y auscultación pulmonar directa con fonendoscopio. Estos exámentes se efectuaron al momento de ałta, al mes siguiente y a los 3, 6,12 y 18 meses de vida.

\section{RESULTADOS}

Durante el periodo en estudio. 54 neonatos requirieron ventilación mecánica, 33 eran varones y también 33 (61\%) habían nacido en la maternidad del hospital Guillermo Grant Benavente de Concepción: los restantes en diferentes hospitales de la Octava Región. Eñ la serie habian 42 recién nacidos pretérmino $(77,8 \%)$ y 12 de término. Respecto al peso de nacimiento, 40 pesaron menos de $2.500 \mathrm{~g}(74,1 \%)$, destacando 7 con peso inferior a $1.000 \mathrm{~g}$ (Tabla $\mathrm{l}$ ). Según cl re. cuento de Apgar en el primer minuto de vida 37 recién nacidos sufrieron asfixia neonatal $(68,4 \%)$, en 22, esta fue grave. A los 5 minutos de vida, 7 recién nacidos tenían recuentos menores o iguales a 3 .

Tabla 1.

Distribución y letalidad según peso de nacimiento en $\$ 4$ recién racidos sometidos a ventilación mecánica.

\begin{tabular}{lcccc}
\hline & Pacicntes & & \multicolumn{2}{c}{ Fallecidos } \\
\cline { 2 - 4 } Peso $(\mathrm{g})$ & $(\mathrm{n})$ & & $(\mathrm{n})$ & $(\%)$ \\
\hline $500-1000$ & 7 & 5 & 71,4 \\
$1001-1500$ & 12 & 8 & 66,6 \\
$1501-2000$ & 12 & 8 & 66,6 \\
$2001-2500$ & 9 & 3 & 33,3 \\
$2501-3000$ & 6 & 4 & 66,6 \\
$3001-3500$ & 4 & 3 & 75,0 \\
3500 o más & 4 & 1 & 25,0 \\
\hline Total & 54 & 32 & 59,3 \\
\hline
\end{tabular}

Las afecciones que motivaron el uso de ventilación mecánica fueron individualizadas en la Tabla 2. La más frecuente fue la membrana hialina (46,3\%), seguida por la asfixia grave. $(25,9 \%)$, el síndrome de aspiración de meconio y la bronconeumonia connatal.

La duración de la ventilación mecánica fue de 5 días menos en 39 casos, 6 a 11 días en 10 pacientes y 12 o más días en $5:$ en 2 de estos últimos duró 53 y 56 días respectivamente y ambos sobrevivieron.

Complicaciones se observaron en 31 casos $(57,4 \%)$. De éstas, las más frecuentes fueron extubación accidental, atelectasia, septicemia, bronconeumonia y hemorragia intracraneana. El ductus persistente se observó en 19,3\% y neumo. tórax, en el $16,1 \%$ de los pacientes (Tabla 3).

Tabla 2

Distribución y letalidad según causas que motivaron la ventilación mecánica en 54 recién nacidos

\begin{tabular}{|c|c|c|c|}
\hline \multirow[b]{2}{*}{ Afección } & \multirow{2}{*}{$\frac{\text { Pacicntes }}{(n)}$} & \multicolumn{2}{|c|}{ Fallecidos } \\
\hline & & (n) & $(\%)$ \\
\hline Membrana hialinis & 25 & 15 & 60,0 \\
\hline $\begin{array}{l}\text { Asfixia neonatal } \\
\text { grave }\end{array}$ & 14 & 10 & 71,4 \\
\hline $\begin{array}{l}\text { Aspiración de } \\
\text { meconio }\end{array}$ & 4 & $y$ & 25,0 \\
\hline $\begin{array}{l}\text { Bronconeumonia } \\
\text { connatal }\end{array}$ & 4 & 2 & 50,0 \\
\hline Septicemia & 2 & 2 & 100,0 \\
\hline $\begin{array}{l}\text { Hemorragia } \\
\text { intracraneana }\end{array}$ & 1 & 1 & 100,0 \\
\hline $\begin{array}{l}\text { Hemorragia } \\
\text { pulmonar }\end{array}$ & 」 & 0 & 0,0 \\
\hline $\begin{array}{l}\text { Hernia } \\
\text { diafragmática }\end{array}$ & 1 & 0 & 0.0 \\
\hline $\begin{array}{l}\text { Meningitis } \\
\text { purulenta }\end{array}$ & 1 & 1 & 100,0 \\
\hline $\begin{array}{l}\text { Circulación fetal } \\
\text { per sistente }\end{array}$ & 1 & 1 & 100,0 \\
\hline Total & 54 & 32 & 59,3 \\
\hline
\end{tabular}

Tabla 3

Identificación y frecuencia de las complicaciones ocurridas en $31 / 54$ recién nacidos sometidos a ventilación mecánici.

\begin{tabular}{lcc}
\hline Tipo de & n & $\%$ \\
\hline Complicación & 14 & 45,2 \\
Extubución & 12 & 38,7 \\
Atelectasia & 11 & 35,5 \\
Septicemia & & \\
Hemorragia & 8 & 25,8 \\
intracrancana & 8 & 25,8 \\
Bronconeumonia & 5 & 16,1 \\
Neumotorax & & 6,5 \\
Enfisema & 2 & 19,3 \\
intersticial & 6 & 6,5 \\
Ductus persistente & & 3,2 \\
Obstrucción tubo & 2 & \\
endotraquea] & 1 & \\
Traumatismo & & \\
de lar inge & & \\
\hline \hline
\end{tabular}

Fallecieron 32 de los 54 recién nacidos, con una letalidad global de 59,3\%. La principal causa de muerte fue la infección grave (septicemia y/o meningitis, bronconeumonia) en el $43,7 \%$, seguido de la hemorragid intracraneana $(21,9 \%)$, membrana hialina $y$ asfixia neonatal grave $(12,5 \%)$

La letalidad según peso de nacimiento fue mayor en los neonatos que pesaron entre 3.000 y 
$3.500 \mathrm{~g}, \mathrm{y}$ en los menores de $1.000 \mathrm{~g}$, con un 75 y $71,4 \%$ respectivamente. La de los RN de muy bajo peso de nacimiento (menos de $1.500 \mathrm{~g}$ ) fue de $68,4 \%$ (Tabla 1 ).

A1 analizar la letalidad por causas, fallecieron todos los recién nacidos que sufrieron septicemia, meningitis, hemorragia intracraneana y circula. ción fetal persistente. Los neonatos con asfixia neonatal grave tuvieron una elevada letalidad $(71,4 \%)$. En los nifios con membrana hialina esta fue de $60 \%$ y en los con bronconeumonia $50 \%$ (Tabla 2). La necropsia realizada en $16 / 32$ pacientes fallecidos mostró buena correlación clínica y anatomopatológica $(87,5 \%)$.

De 22 sobrevivientes, dos fallecieron en el periodo de lactante: uno a la edad de 3 meses, por desnutrición secundaria, atresia esofágica operada, con gastrostomía y esofagostomia de difícil manejo nutricional y otro al mes y 20 días de edad, debido a tetralogia de Fallot extrema cuya corrección quirúrgica no fue posible. Los 20 niños restantes fueron seguidos por períodos de 1 a 17 meses: el fondo de ojo se hizo en 15 de ellos $y$ en todos ha sido nomal. El examen neurológico se realizó en los 20 , reveló alteraciones neuro. lógicas leves en dos pacientes a los 3 y 5 meses y severas en uno con parálisis cerebral $y$ displasia broncopulmonar, que ha motivado 2 hospitalizaciones por bronconeumonia de difícil manejo, en los primeros cinco meses de vida; este último recién nacido permaneció 22 días de ventilación mecánica, con Fi02 mayor o igual a 0,60 por 16 días, debido a una membrana hialina complicada con bronconeumonia, El examen pulmonar fue normal en los 19 niños restantes, sin embargo dos de ellos han presentado un episodio cada uno de bronconeumonia de fácil control.

\section{COMENTARIO}

La membrana híalina es también en esta serie la afección neonatal que con más frecuencia requiere ventilación mecánica $8,9,16,19,23,24$, 25,26 , correspondiendo el mayor porcentaje de casos a neona tos pretérminos, fundamentalmente niños de muy bajo peso de nacimiento $27,28,29$. La duración de la VM con mayor frecuencia menor de 5 días, sugiere que una vez mejorado el problema agudo se puede discontinuar este tipo de procedimiento ${ }^{26,30,31}$.

De las complicaciones observadas, ocuparon el primer lugar los problemas derivados de] tubo endotraqueal, coincidiendo, en general, con la experiencia nacional ${ }^{32}, 33,34,35$. Cabe destacar el número importante de complicaciones por infecciones graves $29,32,33$ y el bajo porcentaje de lesiones con escape de aire (neumotórax y enf isemia intersticial) ${ }^{23}, 28,32,33,35$. Este último resultado favorable puede deberse, en parte, al uso de tiempos inspiratorios cortos, (no mayores de 0,5 segundos) y a la reducción precoz de las presiones inspiratorias altas ${ }^{13}, 16,36,37$. Las frecuencias de hemorragia intracraneana $y$ ductus persistente fueron similares a los señalados en diversas publicaciones $8,9,20,23,28,29$, 32,33 .

No en todos los pacientes conectados a VM se midieron gases en sangre arterial antes del pro. cedimiento, pues debido a la extrema gravedad de su problema muchos fueron ventilados desde los primeros minutos de vida (asfixia perinatal con depresión severa, o paro respiratorio que no respondió a la ventilación manual con ambú). Sin embargo, en los niños en que se practicó el examen la indicación de VM se ajustó a la siguiente pauta: requerimientos de $\mathrm{Fi} 22$ mayores o iguales a 0,7 para mantener la $\mathrm{Pa} 02$ sobre $50 \mathrm{~mm} \mathrm{Hg} \mathrm{y;} \mathrm{PaCO} 2$ mayor de $60 \mathrm{~mm} \mathrm{Hg}$ con un $\mathrm{pH}$ bajo 7,20 en dos exámenes de sangre arterial consecutivos.

El criterio para iniciar ventilación con presión positiva intermitente (IPPV) habitualmente se basa en la alteración de la presión de los gases en sangre arterial ${ }^{13,14,16}$. Sin embargo, hay limi. taciones en la interpretación de los exámenes respectivos ${ }^{13,16}$. La decisión de usar IPPV debe estar basada en un balance de los beneficios y efectos deletéreos del tratamiento. La incidencia y severidad de complicaciones varía de una unidad de cuidado intensivo neonatal a otra, dependiendo de la experiencia y número de personal disponible, del tipo de ventiladores empleados $y$ la dotación de equipos ${ }^{16,37}$. De esta manera, un criterio uniforme para iniciar IPPV no puede ser aplicado en forma rígida y absoluta, siendo necesario que cada unidad desarrolle su propio criterio, basado en sus experiencias y resultados.

La letalidad global de esta muestra concuerda con lo publicado en la literatura nacional29, 32 , 33,34 , pero es algo mayor que en el extranjero ${ }^{8}$, 9, 16, 23, 27. La letalidad entre los recién nacidos de muy bajo peso de nacimiento fue similar a ta observada en otros países ${ }^{9}, 20,27$. 28. No obstante destaca la alta frecuencia de fallecidos entre niños de 3.000 a $3.500 \mathrm{~g}$, en que hubo gran frecuencia de infecciones graves. La letalidad por causas fue mayor que en otras publicaciones para membrana hialina $9,9,23,26$. $29,32,33$, septicemia y meningitis ${ }^{19}$. La elevada letalidad para la membrana híalina se debió a complicaciones como hemorragia intracraneana y septicemia que afectaron a los RN cuando mejoraban de su enfermedad pulmonar, hecho que fue corroborado por la necropsia.

El seguimiento, aunque aún es breve (18 meses), mostró una incidencia de alteraciones 
neurológicas semejante a la de otros países $19,28,39$. Cabe destacar el bajo porcentaje (10\%) de infecciones respiratorias bajas en los neonatos sobrevivientes ${ }^{19}, 26,28,39,40$ y la ausencia de casos con fibroplasia retrolental ${ }^{19}$, $2 \mathrm{a}, 38$

\section{RESUMEN}

Se analizaron los registros de 54 recién nacidos tratados con ventilación mecánica en un periodo de 17 meses.

La causa más frecuente de empleo de asistencia respiratoria fue la membrana hialina $(46,3 \%)$, seguida por la asfixia neonatal grave $(25,9 \%)$. Se observaron complicaciones en $57,4 \%$ de los casos tratados siendo las más frecuentes las extubaciones, las atelactasias e infecciones severas. La letalidad global fue de $59,3 \%$. La causa más frecuente de muerte fue la membrana hialina $(60 \%)$. En los neonatos bajo $1000 \mathrm{~g}$ esta causó $71,4 \%$ de los fallecimientos. En 22 sobrevivientes, seguidos por 1 a 18 meses con exámenes oftalmológicos, pulmonares y neurológicos, no se encontraron alteraciones del fondo de ojo, pero un RN tenía evidencia de displasia broncopulmonar y 3 de daño neurológico, que en un caso fue severo.

\section{REFERENCIAS}

1. Tapia J. y Tapia R.: Panorama de la mortalidad en la niñez en la Octava Región, 1960-1980. Rey. Chil, Pediatr. 54: 192, 1983.

2. Vargos N.; Carretero A. y Pefla $C$.: Variación de algunos factores de riesgo de la mortalidad infantil en Chile entre 1971 y 1980 . Rev. Chil. Pediatr. 54: 41,1983 .

3 Kaempfer $A$, y Medina $E$.: La salud infantil en Chile durante la década del setenta. Rev. Chil. Pediatr. 54: 468, 1982.

4. Toucher E.: Chile: mortalidad desde 1955 y 1975. Tendencias y causas. CELADE, Agosto, 1978.

5. Toucher E.: La mortalidad infantil en Chile. Notas de Población. CELADE, Agosto, 1979.

6. Lee K.: Paneth N.: Garner L. et al: Nconatal mortality: an analysis of the recent improvement in the United States. Am. J. Public Health, 70: 15, 1980 .

7. Schreiner R.: Kusling J.: Evans G. et al: Improved survival of ventilated neonates with modern intensive care. Pediatrics, 66: 1985, 1980.

8. Boyle $R$. and $O h W$. Sindrome de dificultad respiratoria. Clin. Perinatol 2: 283, 1978.

9. Corbet A. and Adams J.: Terapéutica actual de la enfermedad de membrana hialina. Clin. Perinatol 2: $299,1978$.

10. Stavis $R$. and Krouss A.: Complicaciones de cuidado neonatal intensivo. Clin. Perinatol $1: 105,1980$.

11. Daity $W$. and Smith P.: Mechanical ventilation in the newborn. II Curr. Probl. Pediatr., 1: 30, 1971.

12. Sykes M.; McNicol $M$. and Campbell E.: Respiratory failure. Oxford, Blackwell Scientific Publications, 1969.
13. Brady J. and Gregory $G_{*}$. Ventilación asistida. Asistencia del RN de al to riesgo. Ed. Panamericana, 1980.

14. Klaus M.: Fonaroff A. and Martin R.: Problemas respiratorios. Asistencia del RN de atto riesgo. Ed. Panamericana, 1980

15. Newth M: Recognition and manegement of respi. ratory failure. Pediatr. Clin. North Am. 26: 617, 1979.

16. Krauss A.: Assisted ventilation: a critical review. Clin. Perinatol. 7: 61, 1980 .

17. Girling $D$. and Hallidie-Smith $K$.: Persistent ductus arteriosus in ill and premature babies. Arch. Dis. Child., 46: 177, 1971.

18. Kiftermen J.; Edmunds L.: Gregoy G. et al: Patent ductus arteriosus in premature infants: incidence, relation to pulmonary disease and menegement. $N$. Engl. J. Med., 287: 473, 1972.

19. Kamper J.: Prognosis of neonates with symptomatic respiratory insufficiency surviving with the aid of ventilatory therapy. Acta Pediatr. Scand. 67: 53, 1978 .

20. De Lemos R. y Tomasovic J.: Efectos de la ventilación mecánica a presión positiva sobre el riego sanguíneo cerebral en el neonato. Clin. Perinatol. 2: 391, 1978.

21. Katfan $M .:$ Long-term sequelae of respiratory illness in infancy and chilhood. Pediatr. Clin. North Am. 26: 525, 1979.

22. Cootes A.; Bergsieisson H.; Desmond K.; Outerbridge $E$. and Beaudry P.: long-term pulmonary sequelae of prematwe birth with and without idiopatic respiratory distress syndrome. J. Pediatr. 90: $611,1977$.

23. Reynolds $E$. and Taghizadih A.: Improved prognosis of in fants mechanically ventilated for hyaline membrane disease. A rch. Dis. Child., 49: 505, 1974.

24. Gerard P.; Fox W.; Outerbridge E.; Beaudry P. and Stern $L$ : : Early versus late introduction of continuos negative pressure in the menegement of the idiopatic respiratory distress syndrome. J. Pediatr. 87: 591,1975

25. Mockrin L, and Bancalari E.: Early versus delayed initiation of continuos negative pressure in infants with hyaline membrane disease. J. Pedjatr. 87:596. 1975.

26. Stahtman M.; Hedval G.; Lindstrom D. and Snell L.: Papel de la membrana hialina en el desarrollo de anomalías pulmonares en la fase posterior de la infencia. Pediatrics, 13: 572, 1982.

27. Cohen R.; Stevenson D.; Malachowski N.; Ariagno R.; Kimble K.; Hopper H.; Johnson J.; Velend $K$ and Sunshine P.: Resultados favorables de las unidades de cuidados intensivos neonatales para los recién nacidos de muy bajo peso. Pediatrics, 13: 381,1982 .

28. Fitzhardinge P.: Pape $\boldsymbol{K}_{\text {.; }}$ Arstikaitis M.; Boyle M.; Ashby S.: Rowley A.; Netley $C$. and Swyer P.: Mechanical ventilation of infants of less than $1501 \mathrm{~g}$ birth weight: Health, growth and neurological sequelae. J. Pediatr. 88: $531,1976$.

29. Corrés E.; Guzmán M. y Azócar R.: Evaluación de un año de terapia ventilatoria en unidad de recién nacidos. Maternidad Barros Luco-Trudeau. Resumen temas libres XIV Congreso Chileno Pediatría, Puerto Montt: 118, 1982.

30. Daily w.; Meyer H.: Sunshine P. and Smith P.: Mechanical ventilation of newbors infants. II] Historical comments and development of a scoring system for selection of infants. Anesthesiology, 34: $119,19 ? 1$. 
31. Barr $P .:$ Weaning very low birthweight infants from mechanical ventilation using intermitent mandatory ventilation and theophylline. Arch. Dis. Child. 53: $598,1978$.

32. Moreno A.; Alarcón J. and Saluo H.t Experiencia de 20 meses en ventilación en una unidad de tratamiento intensivo neonatal. Resumen temas libres. XIV Congreso Chileno Pediatria, Puerto Montt: $105,1982$.

33. Moreno A.; Alarcón J.; Beca J.; Wilson J. and Salvo H.: Ventilación mecánica en recién nacido. Experiencia de tres años. Resumen temas libres. $X$ Jornadas Nacionales Pediatría, Vüna del Mar: 61, 1983.

34. Cerda M.; Saavedrz O.; Aspitlaga M., Mesa $T$. et al. Ventilación mecánica en insuficiencia respiratoria de origen pulmonar, Rev. Chil. Pediat. $55: 25$, 1984 .

35. Bancalori E. and Berlín J.: Aspitación de meconio y otros problemas asfixticos. Clin. Perinatol. 2: 317. 1978.

36. Boros $S$. and Orgill $A$.: Mortality and morbility associated with pressure - and volumen limited infant ventilation. Am. J. Dis. Chil. 132: 865, 1978.

37. Simbruner G.: Popow $C$.; Baum $M$. and Gregory G.: Respiradores para el neonato. Clin. Perinatol. I: $215,1983$.

38. Kamper $J$.: Long-term prognosis of infants with severe idiopathic respiratory distress syndrome. I. Neurological and mental outcome. Acta Pediatr. Scand. 67: 61, 1978 .

39. Fitzhardinge $P$.: Estudio de vigilancia en neonatos tratados con ventilación mecánica. Clín. Perinatol. 2: 449,1978 .

40. Kamper $J_{\text {: }}$ Long-term prognosis of infants with severe idiopathic respiratory distress syndrome. Il Cardiopulmonary outcome. Acta Pediatr. Scand. 67: 71, 1978 . 